\title{
Insula and Striatum Mediate the Default Bias
}

\author{
Rongjun Yu, ${ }^{1}$ Dean Mobbs, ${ }^{1}$ Ben Seymour, ${ }^{2}$ and Andrew J. Calder ${ }^{1}$ \\ ${ }^{1}$ Medical Research Council Cognition and Brain Sciences Unit, Cambridge CB2 7EF, United Kingdom, and ${ }^{2}$ Wellcome Trust Centre for Neuroimaging, \\ University College London, London WC1N 3BG, United Kingdom
}

Humans are creatures of routine and habit. When faced with situations in which a default option is available, people show a consistent tendency to stick with the default. Why this occurs is unclear. To elucidate its neural basis, we used a novel gambling task in conjunction with functional magnetic resonance imaging. Behavioral results revealed that participants were more likely to choose the default card and felt enhanced emotional responses to outcomes after making the decision to switch. We show that increased tendency to switch away from the default during the decision phase was associated with decreased activity in the anterior insula; activation in this same area in reaction to "switching away from the default and losing" was positively related with experienced frustration. In contrast, decisions to choose the default engaged the ventral striatum, the same reward area as seen in winning. Our findings highlight aversive processes in the insula as underlying the default bias and suggest that choosing the default may be rewarding in itself.

\section{Introduction}

Whether it is selecting weekly lottery numbers or renewal of an annual insurance policy, we are repeatedly faced with situations in which we must accept the default option or switch to an alternative. While such decisions often incur a gain or loss of money or other commodity, studies have shown that even when these are equated, an increased preference for the default is found (Samuelson and Zeckhauser, 1988; Johnson and Goldstein, 2003). Yet, why this occurs is unclear.

Several explanations have been proposed. For example, in some circumstances defaults may be seen as optimal or recommended options, such as buying rental car insurance, and so people are more likely to select them. Alternatively, individuals may be reluctant to switch away from defaults because switching involves greater mental or physical effort (Anderson, 2003). However, even when effort is equated, there is evidence that anticipation of loss ("loss aversion") may impel the default preference (Kahneman et al., 1991). This is because although choosing defaults and non-defaults could both result in the same negative outcomes, individuals' subjective evaluation of these two types of aversive outcomes is different. Studies from behavioral economics using hypothetical scenarios show that individuals exhibit more regret when negative outcomes result from atypical actions versus routine actions (Kahneman and Miller, 1986). Thus, individuals are more willing to choose defaults to avoid the enhanced negative emotion associated with choosing nondefaults. Of course, default and non-default options can also be associated with gains. However, the greater emphasis on loss is explained by the proposal that anticipated losses loom larger than anticipated gains (Kahneman et al., 1991).

Received July 20, 2010; revised Aug. 30, 2010; accepted Sept. 13, 2010.

The research was funded by the United Kingdom Medical Research Council (Grant U1055.02.001.00001.01 to A.J.C..). R.Y. is supported by the UK/China Scholarships for Excellence.

Correspondence should be addressed to Rongjun Yu, MRC Cognition and Brain Sciences Unit, 15 Chaucer Road, Cambridge CB2 7EF, United Kingdom. E-mail: ry223@cam.ac.uk or rongjun.yu@gmail.com.

DOI:10.1523/JNEUROSCI.3772-10.2010

Copyright $\odot 2010$ the authors $\quad 0270-6474 / 10 / 3014702-06 \$ 15.00 / 0$
It remains unknown, however, how the act of choosing a nondefault option modulates the neurophysiological representation of the experienced hedonic value of a stimulus, as well as our emotional response to it. Moreover, previous studies cannot tell us if it is aversive to switch away from or even rewarding to stay with the default. For example, one might predict that the act of switching (before win/loss outcome) is associated with brain areas such as the insula, which are involved in anticipation and receipt of aversive stimuli (Clark et al., 2008; Preuschoff et al., 2008). Conversely, it is possible that sticking with the default is associated with reward systems (e.g., ventral striatum). To address these questions directly, we used functional magnetic resonance imaging ( $\mathrm{fMRI}$ ) in conjunction with a novel gambling task that comprised two phases. During a decision phase, one gambling card was assigned to the participant and they had the option to either stay with this default card or switch to another; the following outcome phase indicated the value of the cards and the amount won or lost. Our design allowed us to identify the neural correlates of switch and stay choices and to test the hypothesis that the neurobiological representation of an outcome's subjective value is exaggerated after switching away from the default. Further, our design controlled the effort associated with deciding to switch or stay in that both actions required an active buttonpress response.

\section{Materials and Methods}

\section{Experiment 1}

Participants. Eighteen healthy volunteers (8 males; mean age \pm SD, $22.4 \pm 3.2)$ participated in return for payment. All participants were right handed, were fluent speakers of English, and were screened for psychiatric or neurological problems. The study was authorized by the National Health Service Local Research Ethics Committee for Cambridge. All participants gave written, informed consent and were informed of their right to discontinue participation at any time.

Experimental paradigm. On each trial, participants were first presented with the value of two possible outcomes, a potential win and a potential loss for $4 \mathrm{~s}$. Following this, they were then presented with two cards, one of which was the default card assigned to the participant, highlighted in 

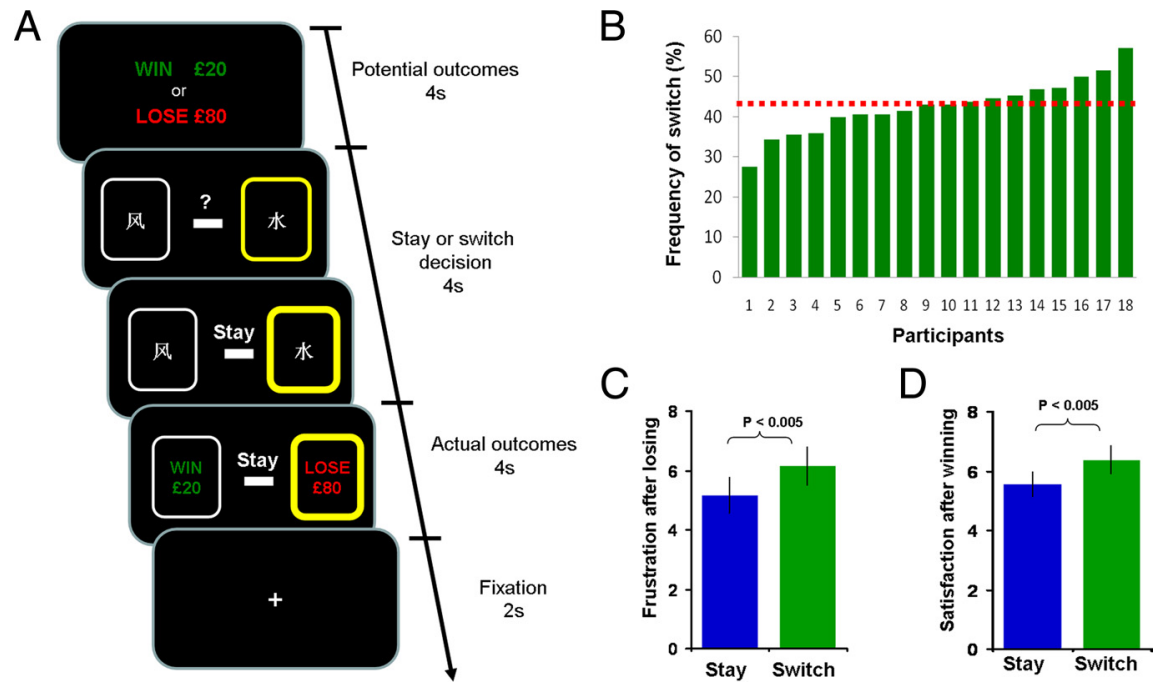

echo time (TE), $25 \mathrm{~ms}$; flip angle $=90^{\circ}$; field of view, $224 \mathrm{~mm}$; voxel size, $3 \times 3 \times 3 \mathrm{~mm}$ ). To allow for equilibration effects, the first three volumes were discarded. T1-weighted structural images were acquired at a resolution of $1 \times 1 \times 1 \mathrm{~mm}$.

Image preprocessing. SPM5 software (www. fil.ion.ucl.ac.uk/spm/) was used for data analysis. The EPI images were sync interpolated in time for correction of slice-timing differences and realigned to the first scan by rigid-body transformations to correct for head movements. Field maps were estimated from the phase difference between the images acquired at the short and long TE and unwrapped, using the FieldMap toolbox. Field map and EPI parameters were used to establish voxel displacements in the EPI image. Application of the inverse displacement to the EPI images served in the correction of distortions. Utilizing linear and nonlinear transformations, and smoothing with a Gaussian kernel of full-width-halfmaximum $8 \mathrm{~mm}$, EPI and structural images were coregistered and normalized to the T1 standard template in Montreal Neurological Institute (MNI) space (MNI-International Consortium for Brain Mapping). Global yellow (Fig. 1A). Participants were required to choose either to stay with the default card or switch to the other card by pressing the key that corresponded to the position of the card, within $4 \mathrm{~s}$. Finally, the outcomes associated with both cards were presented for $4 \mathrm{~s}$. Unknown to participants, the outcomes were predetermined so that they lost $50 \%$ of the time. The magnitude of winning and losing was manipulated independently, each ranging from $\mathfrak{1} 10$ to $£ 80$, in increments of $£ 10$, to maintain participants' interest. The stimulus presentation and response recording was controlled by E-prime. The whole experiment included 128 trials and lasted $\sim 28 \mathrm{~min}$. Before the experiment, participants were told that the total amount of money they accumulated in the experiment would be exchanged into real monetary reward at a certain exchange rate. At the end of the experiment, all participants were rewarded $\mathfrak{E} 10$ bonus in addition to the standard payment for participation.

Postscan questionnaires. After the end of the experiment, participants were asked to indicate how frustrated they felt when they lost after switching and when they lost after staying with the default on a 10 point analog Likert scale ( $1=$ not at all; $10=$ very intensely). For example, we used questions like "How frustrated did you feel in the following situations?" and presented two snapshot images of the two types of losing outcomes (losses after switching and losses after staying) underneath the questions. They were also asked to indicate how satisfied they felt when they won after switching and when they won after staying.

Computational analysis. The standard temporal difference (TD) model was used to examine the possible learning effect. In the TD model, the value $V_{i}$ for each gambling card is updated whenever it is chosen. Specifically, whenever gambling card $i$ was chosen, its predicted value $V_{i}$ was updated in the direction of the obtained reward $r$ using a delta rule with learning-rate parameter $\alpha: V_{i}=V_{i}+\alpha\left(r-V_{i}\right)$. The probability of choosing option $i$ was taken to follow a "softmax" distribution in its value (i.e., $p_{2}=\exp \left(t V_{2}\right) /\left[\exp \left(t V_{2}\right)+\exp \left(t V_{1}\right)\right]$ ). Free parameters (learning rate $\alpha$, softmax inverse temperature $t$ ) were selected per subject to optimize the likelihood of that subject's sequence of choices (i.e., the product of the probability of each choice, defined as above, over all trials). As measures of the quality of model fit, we compare the negative log likelihoods of the data with the negative log likelihoods assuming purely random choices.

Image acquisition. MRI scanning was conducted at the Medical Research Council Cognition and Brain Sciences Unit on a 3 tesla Tim Trio Magnetic Resonance Imaging scanner (Siemens) using a head coil gradient set. Whole-brain data were acquired with echo planar $\mathrm{T} 2{ }^{*}$-weighted imaging (EPI), sensitive to blood oxygenation level-dependent signal contrast [48 sagittal slices, $3 \mathrm{~mm}$ thickness; repetition time, $2400 \mathrm{~ms}$; changes were removed by proportional scaling and high-pass temporal filtering with a cutoff of $128 \mathrm{~s}$ to remove low-frequency drifts in signal.

Statistical analysis. After preprocessing, statistical analysis was performed using the general linear model. Analysis was performed to establish each participant's voxelwise activation during the stay or switch decision epochs and actual outcome epochs. Activated voxels in each experimental context were identified using an event-related statistical model representing the six experimental conditions (stay choices, switch choices, losses after switch, losses after stay, wins after switch, and wins after stay), convolved with a canonical hemodynamic response function and mean corrected. Six head-motion parameters defined by the realignment were added to the model as regressors of no interest. Multiple linear regression was then run to generate parameter estimates for each regressor at every voxel. A random-effects analysis (one-sample $t$ test) was performed to analyze data at a group level. All conjunction analyses were performed using the SPM 5 conjunction null function (Friston et al. 2005). Small volume correction ( $\mathrm{svc}$ ) was used on a priori regions of interest including the following: (1) the anterior insula, which was defined by restricting the structural template for the insula derived from automated anatomical labeling (Tzourio-Mazoyer et al., 2002) to the region anterior to the anterior commissure plane (i.e., $y>0$ ); (2) ventral striatum-10 $\mathrm{mm}$ sphere at $\pm 14,10$, and -10 (O’Doherty et al., 2004); and (3) Caudate (dorsal striatum), defined by the corresponding automated anatomical labeling mask. Activations in other areas are reported if they survive $p<0.001$ uncorrected, cluster size $k>10$. For display purposes, all images are depicted at $p<0.005$.

\section{Experiment 2}

One concern regarding experiment 1 is whether emotion ratings obtained after the experiment provide a reliable estimate of emotions experienced during the task. To address this, we conducted a behavioral study recording both trial-by-trial emotion ratings and postexperiment ratings.

Participants. Fifteen healthy volunteers (6 males; mean age \pm SD, $24.6 \pm 2.7)$ participated in return for payment. All participants were right handed, were fluent speakers of English, and were screened for psychiatric or neurological problems. The study was authorized by the National Health Service Local Research Ethics Committee for Cambridge. All participants gave written, informed consent and were informed of their right to discontinue participation at any time.

Experimental paradigm. We used the same experimental task but with two modifications: first, after each win or loss outcome participants were required to indicate how frustrated or how satisfied they felt using a 10 
point visual analog scale $(1=$ not at all, $10=$ very intensely $)$. Second, the task was self-paced, and participants pressed a key when they finished each set of ratings to proceed to the next trial. The whole experiment included 64 trials.

\section{Results \\ Experiment 1 \\ Behavioral data}

Participants revealed a preference for the default card (57.2\% stay vs $42.8 \%$ switch, $t_{17}=4.53, p<0.001$ ) (Fig. $1 B$ ). However, reaction times for switch and stay were comparable (mean $\pm \mathrm{SD}$, $1086 \pm 471 \mathrm{~ms}$ for switch vs $1050 \pm 411 \mathrm{~ms}$ for stay, $t_{17}<1$ ), suggesting that the difference in mental effort involved in switching was negligible.

Subjective emotion ratings after the experiment showed that participants felt stronger frustration for losses after switch $\left(\mathrm{L}_{\text {switch }}\right)$ than after stay $\left(\mathrm{L}_{\text {stay }}\right)\left(t_{17}=3.43, p<0.005\right)$ (Fig. 1C) and expressed more satisfaction for wins after switch $\left(\mathrm{W}_{\text {switch }}\right)$ than after stay $\left(\mathrm{W}_{\text {stay }}\right)$ $\left(t_{17}=3.39, p<0.005\right)$ (Fig. $\left.1 D\right)$.

We also examined the possible effects of feedback from the immediately preceding trial on stay or switch choices. We did not find a significant main effect of previous winning or losing outcome $\left(F_{(1,17)}=0.33, p>0.5\right)$ nor a main effect of previous staying or switching choices $\left(F_{(1,17)}=0.74, p>0.4\right)$. No interaction between these two factors was found $\left(F_{(1,17)}=1.58, p>0.2\right)$. Using the standard TD model, we did not find a significant learning effect. The errors resulting from the TD model did not significantly differ from the errors resulting from the model assuming purely random choices (i.e., choosing to stay with 50\% chance) $\left(t_{17}=1.84, p>0.05\right)$.

\section{fMRI results}

Decision phase: switch versus stay. During the decision phase (i.e., $4 \mathrm{~s}$ from the onset of the default and non-default cards to their offset), switch choices (relative to stay choices) activated the right anterior insula [40, 12, -10 ; peak $z=4.35$; $p<0.05 \mathrm{svc}$ ) (Fig. $2 A)$. Further, for the switch minus stay contrast, increased frequency of switching was significantly correlated with decreased activity in right anterior insula $[40,14,2$; peak $z=3.05 ; p<0.05$ $\mathrm{svc}]$ and increased activity in left caudate $[-18,22,12$; peak $z=$ 3.62; $p<0.05 \mathrm{svc}$ ] (Fig. $2 A, C$ ). Our data suggest that the relative balance of insula and caudate activity in the decision phase may be associated with participants' decisions to stay or switch.

In contrast, stay choices (relative to switch) activated the left ventral striatum $(-18,6,-6$; peak $z=3.08$; $p<0.05 \mathrm{svc}$ ) (Fig. $2 B)$, a region implicated in reward, and the same region activated by winning during the outcome phase (see below).

We also modeled the decision phase using the epoch from the onset of the default and non-default cards to the time participants responded. Similar response patterns were found. Switch choices (relative to stay choices) activated the right anterior insula (42, 18, -6 ; peak $z=2.89 ; p=0.05 \mathrm{svc}$ ) (Fig. $2 A$ ) and stay choices (relative to switch) activated the left ventral striatum $(-18,8,-6$; peak $z=2.64 ; p=0.06 \mathrm{svc}$ ) (Fig. $2 B$ ). Further, for the switch minus stay contrast, increased frequency of switching was significantly correlated with decreased activity in right anterior insula $(38,20,6$; peak $z=3.03$; $p<0.05 \mathrm{svc})$ and increased activity in left caudate $(-18,22,12$; peak $z=3.76$; $p<0.05 \mathrm{svc})$.

To examine the effect of previous outcomes (win or loss) on the immediate subsequent stay or switch decisions, we estimated another model in which we used four regressors (previous win-switch, previous loss-switch, previous win-stay, and previous loss-stay) instead of the two regressors (switch/stay). No significant effects were observed in predicted areas of interest at $p<0.001$, in the following
A

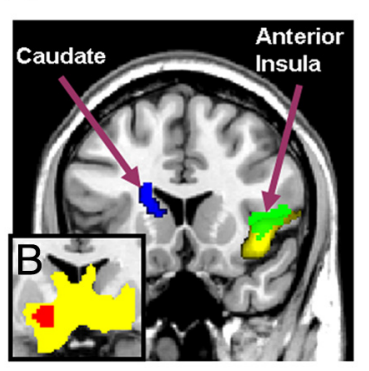

D

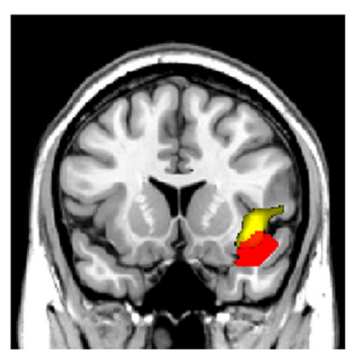

C

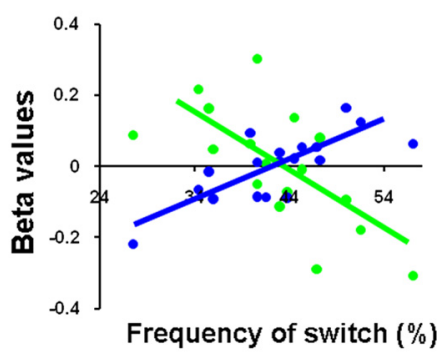

E

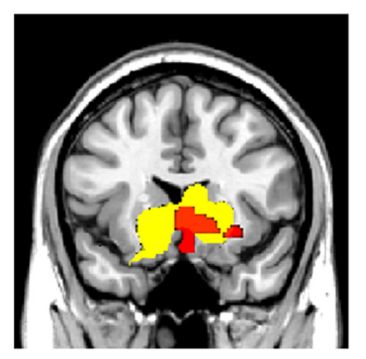

Figure 2. $\quad \boldsymbol{A}, \boldsymbol{B}$, Right anterior insula (yellow) activated by switch versus stay choices $(\boldsymbol{A})$, and left ventral striatum (red) activated by the reverse comparison (stay versus switch) (B), overlapped with striatum (yellow) activity responding to winning outcomes. $\boldsymbol{A}, \boldsymbol{C}$, For switch versus stay comparison, insula (green) activity was negatively correlated with the tendency to switch, while caudate (blue) activity was positively associated with switch frequency. D, Significant activity associated with the residual frustration scores (i.e., frustration for $L_{\text {switch }}$ minus frustration for $\mathrm{L}_{\text {stay }}$ ) in right anterior insula (red) for the comparison of $\mathrm{L}_{\text {switch }}$ versus $\mathrm{L}_{\text {stay }}$ outcomes and significant activity in the right anterior insula (yellow) associated with switch versus stay decisions. $\boldsymbol{E}$, Significant activity associated with the residual satisfaction scores in ventral striatum (red) for the comparison of $W_{\text {switch }}$ versus $W_{\text {stay }}$ and significant activity in bilateral ventral striatum (yellow) associated with winning versus losing. All activations are significant at $p<$ 0.05 , small volume corrected.

contrasts: previous win-switch minus previous loss-switch; previous loss-switch minus previous win-switch; previous win-stay minus previous loss-stay; previous loss-stay minus previous win-stay; and the interactions (current switch/stay choices modulated by previous win/loss). Outside regions of interests, the postcentral gyrus $(x=$ $-60, y=0, z=22$; peak $z=4.16 ; p<0.001$, uncorrected) showed an effect for the interaction (i.e., [switch - stay $\times$ win - loss]), but no other effects were found.

Outcome phase: winning versus losing. For the outcome phase, we found that winning outcome versus losing activated bilateral ventral striatum (left: $-10,12,-12$; peak $z=4.34 ; p<0.001 \mathrm{svc;}$ right: $10,14,-6$; peak $z=4.05, p<0.002 \mathrm{svc}$ ) (Fig. $2 B$ ), ventromedial prefrontal cortex $(-8,46,-2$; peak $z=3.88 ; p<0.0005$ uncorrected), bilateral orbitofrontal cortex (left: $-42,42,-16$; peak $z=4.15 ; p<0.0005$ uncorrected; right: 44, 44, -6 ; peak $z=$ 3.93; $p<0.0005$ uncorrected), and bilateral premotor cortex (left: $-14,40,50$; peak $z=3.74 ; p<0.0005$ uncorrected; right: $24,32,48$; peak $z=4.05 ; p<0.0005$ uncorrected). No significant activation was found for the reverse contrast (i.e., losing vs winning), which is consistent with previous findings (O'Doherty et al., 2004; Tom et al., 2007).

Conjunction: stay versus switch and winning versus losing. A conjunction analysis between stay minus switch decisions in the decision phase and winning minus losing in the outcome phase demonstrated that a region of left ventral striatum $(-18,6,-6$; peak $z=3.08 ; p<0.05 \mathrm{svc}$ ) was activated in both conditions (Fig. $2 B$ ). Thus, our data suggest that choosing to stay with the defaults might be rewarding in itself. This may be because it avoids the 
aversive quality of switching, produced by the combined effects of outcomes after switching having an enhanced emotional quality and the established observation that anticipated losses loom larger than anticipated gains (Kahneman et al., 1991).

The reverse conjunction between switch versus stay and losing versus winning did not produce a significant result. However, this can be explained by the absence of significant activation for the losing versus winning contrast.

Outcome phase: loss after switch versus loss after stay. As discussed, participants found it more frustrating when they lost after switching than when they lost after staying (Fig. 1C). To examine whether the amount of frustration was related to the neural response, we subtracted frustration scores for $\mathrm{L}_{\text {stay }}$ from frustration scores for $\mathrm{L}_{\text {switch }}$ to provide a residual frustration score. A correlation analysis examining the relationship between residual frustration scores and activation for the $\mathrm{L}_{\text {switch }}$ minus $\mathrm{L}_{\text {stay }}$ contrast revealed significant increases in right anterior insula $(42,14,-12$; peak $z=3.69 ; p<0.05 \mathrm{svc}$ ) (Fig. $2 D$ ), anterior cingulate cortex $(10,38,28$; peak $z=3.78 ; p<0.0005$ uncorrected), and bilateral dorsolateral prefrontal cortex (left: $-36,30$, 46; peak $z=3.72$; $p<0.0005$ uncorrected; right: 38, 22, 50; peak $z=4.08$; $p<$ 0.0005 uncorrected). The main group effect of $\mathrm{L}_{\text {switch }}$ minus $\mathrm{L}_{\text {stay }}$ did not identify any brain regions at the a priori threshold. Thus, although the objective value of $\mathrm{L}_{\text {switch }}$ and $\mathrm{L}_{\text {stay }}$ are identical, $\mathrm{L}_{\text {switch }}$ is experienced as more aversive than $\mathrm{L}_{\text {stay }}$, and the degree of aversion is associated with anterior insula activation.

A conjunction analysis between $\mathrm{L}_{\text {switch }}$ minus $\mathrm{L}_{\text {stay }}$ modulated by residual frustration scores and switch minus stay choices (decision phase) confirmed that the right anterior insula $(42,14$, -12 ; peak $z=3.69$; $p<0.05 \mathrm{svc}$ ) was activated in both conditions. Our data suggest that the anticipation of the enhanced frustration to switching and losing at the time of the decision might underlie the loss aversion in the default preference.

Outcome phase: win after switch versus win after stay. Participants found it more rewarding for wins after switching than wins after staying (Fig. 1D). The residual satisfaction scores (satisfaction for $\mathrm{W}_{\text {switch }}$ minus satisfaction for $\mathrm{W}_{\text {stay }}$ ) were significantly correlated with increased activity in right ventral striatum $(10,18$, -6 ; peak $z=3.13$; $p<0.05 \mathrm{svc}$ ) (Fig. $2 E$ ) and ventromedial prefrontal cortex $(12,40,-4$; peak $z=3.80$; $p<0.0005$ uncorrected) for this contrast. The only region engaged by the main group effect of $\mathrm{W}_{\text {switch }}$ minus $\mathrm{W}_{\text {stay }}$ was the occipital cortex $(-16$, $-92,-12$; peak $z=3.32 ; p<0.001$ uncorrected); indicating that the neural responses in the ventral striatum and ventromedial prefrontal cortex were governed by the amount of satisfaction participants experienced. The conjunction analysis between $\mathrm{W}_{\text {switch }}$ minus $\mathrm{W}_{\text {stay }}$ modulated by residual satisfaction scores and winning minus losing outcomes confirmed that the right ventral striatum $(10,18,-6$; peak $z=3.13$; $p<0.05 \mathrm{svc})$ was activated in both conditions. Thus, both the behavioral and neural evidence indicate that switching augments the rewarding experience for positive outcomes.

\section{Experiment 2}

\section{Behavioral data}

Consistent with experiment 1, participants revealed a preference for the default card (58.4\% stay vs $41.6 \%$ switch; $t_{14}=14.61 ; p<$ 0.001 ) and showed comparable reaction times for switch and stay decisions (mean \pm SD: $824 \pm 368 \mathrm{~ms}$ for switch vs $783 \pm 336 \mathrm{~ms}$ for stay; $t_{14}=1.24 ; p>0.2$ ), suggesting that the difference in mental effort involved in switching was negligible.

Subjective emotion ratings after the experiment showed that participants felt stronger frustration for $\mathrm{L}_{\text {switch }}$ than $\mathrm{L}_{\text {stay }}\left(t_{14}=\right.$
6.86; $p<0.001$ ), and expressed more satisfaction for $\mathrm{W}_{\text {switch }}$ than $\mathrm{W}_{\text {stay }}\left(t_{14}=4.03 ; p=0.001\right)$. Similarly, the online emotion ratings also revealed that participants felt stronger frustration for $\mathrm{L}_{\text {switch }}$ than for $\mathrm{L}_{\text {stay }}\left(t_{14}=3.82 ; p<0.005\right)$, and expressed more satisfaction for $\mathrm{W}_{\text {switch }}$ than for $\mathrm{W}_{\text {stay }}\left(t_{14}=4.10 ; p=0.001\right)$.

The residual frustration scores (i.e., frustration for $\mathrm{L}_{\text {switch }} \mathrm{mi}$ nus frustration for $\mathrm{L}_{\text {stay }}$ ) for online emotion ratings were significantly correlated with the residual frustration scores for ratings collected after the experiment $(r=0.68 ; p=0.005)$. The corresponding correlation for the residual satisfaction scores was also significant $(r=0.61 ; p<0.02)$. The results therefore suggest that emotion ratings collected after the experiment provide a valid estimate of emotions experienced during the task.

\section{Discussion}

We investigated the neural basis of the default bias. Although opting to switch in the decision phase (before win/loss outcome) was associated with increased insula activity across all participants, individual differences in frequency of switching showed a negative relationship with insula activation and a positive relationship with caudate activation. Notably, the same insular area showed a positive relationship with frustration after switching away from the default and losing. In contrast, deciding to stay with the default engaged the ventral striatum, a region implicated in reward processing (O’Doherty et al., 2004), including winning outcomes in our current study. In the context of previous data on the function of these regions in motivational processes, our results not only support theories that aversive mechanisms influence decisions to stay with the default but provide new evidence, suggesting that it is rewarding to stay put. These findings were observed despite an equal probability that the default card was associated with win or loss, and controlling for any social factors (e.g., conformity to a default social norm).

The patterns of activation for switch versus stay (insula) and stay versus switch (ventral striatum) contrasts during the decision phase are striking because the objective outcomes associated with these options were equivalent and the effort involved in selecting each was comparable (i.e., both required a button-press response with equivalent execution times). So, what makes these two types of choices different? One contributory factor may arise from the observation that the subjective experience of outcomes after stay and after switch is different and participants may therefore anticipate this. The behavioral results demonstrate that outcomes resulting from choosing the non-default choice generally lead to more intense affective reactions than the same outcomes achieved through selecting the default. A second contributory factor is the established observation that losses loom larger than gains (Kahneman et al., 1991), resulting in an overall bias to avoid intense aversive events associated with switching and losing.

The insula has been implicated in anticipation and receipt of risky, aversive stimuli (Paulus et al., 2003; Preuschoff et al., 2008; Craig, 2009), and insula damage can result in elevated risk taking (Clark et al., 2008). These studies suggest that the insula is involved in generating anticipatory somatic markers of risky, aversive events. Similarly, in our own study, insula activity elicited by switching might represent an aversive somatic state when making switch choices, since these are perceived as more risky than outcomes associated with stay choices for the reasons outlined. Consistent with this, we found that overall switch choices, compared with overall stay choices, resulted in a stronger aversive somatic maker (i.e., insula activation). Similarly, and in line with the observation that insula activity is associated with heightened skin conductance responses (SCRs) (Buchel et al., 1998; Critchley et 
al., 2000), Bechara et al. (1997) found that participants showed more pronounced anticipatory SCRs before choosing from risky than safe decks in the Iowa gambling task. Moreover, consistent with our observation that insula activation to switch minus stay choices in the decision phase was negatively related to the frequency of switch choices, Dunn et al. (2010) showed that the differential SCR between selection of risky and safe card decks was negatively related to the frequency of risky deck selections in a modified Iowa gambling task. A role for the insula area identified in processing risky aversive events is further supported by our observation that $\mathrm{L}_{\text {switch }}$ minus $\mathrm{L}_{\text {stay }}$ events in the outcome phase modulated by residual frustration scores identified the same insula region associated with switch choices in the decision phase. By contrast, staying with the default engaged the same area as winning (ventral striatum) and might be rewarding in itself (Seymour et al., 2004).

Finding that frequency of switching was negatively correlated with right anterior insula and positively correlated with left caudate accords with the idea that default/non-default decision making involves a tradeoff between avoiding high loss $\left(\mathrm{L}_{\text {switch }}\right)$ and seeking high reward $\left(\mathrm{W}_{\text {switch }}\right)$. Thus, individuals who show relatively greater insula and relatively reduced caudate activity at the decision stage might end up choosing to stay with the default more often because for them, the anticipation of loss-after-switching, which is more aversive than loss-after-default-selection as our behavioral data show, outweighs the pleasure of anticipation of winafter-switching. In contrast, relatively greater caudate and relatively reduced insula activity at the decision stage might emphasize the enhanced positive experience associated with switching and winning, encouraging the subject to take the risk and switch. Consistent with this, recent studies have shown increased activation in the caudate nucleus when subjects anticipate or imagine positive future events (Knutson et al., 2001; Sharot et al., 2007, 2009). However, in line with the proposal that anticipated losses loom larger than anticipated wins, the overall group bias is to avoid loss.

Our observation that choosing defaults or non-defaults affected individuals' subjective evaluation of monetary outcomes and patterns of neural activity accords with previous work showing that the experience of an event is highly subjective and can vary substantially depending on the context. For example, fMRI research has consistently demonstrated that ventral striatal responses to the same amount of reward differ depending on the range of possible outcomes from which an outcome was selected (Nieuwenhuis et al., 2005), its relative magnitude in relation to another participant's reward (Fliessbach et al., 2007), and the choice commitment (Sharot et al., 2009). Consistent with these studies, we showed that striatal and insula activity in response to winning and losing outcomes, respectively, varied depending on whether the outcome was achieved through choosing a default or not. Similarly, there is evidence that expectation can profoundly modulate subjective experience of aversive stimuli (e.g., aversive taste, painful stimulation) and associated insula activity (Koyama et al., 2005; Nitschke et al., 2006). Our findings suggest that both aversion/risk-sensitive (e.g., insula) and reward-sensitive (e.g., striatum) brain areas can track the subjective hedonic experience of stimuli in the financial domain, even when their objective values are the same.

Although we propose that emotions play an important role in mediating the default bias, a more precise explanation of why emotional reactions to outcomes after switch are amplified remains unclear. One possibility is that participants feel more responsible for outcomes following switch choices and that responsibility is the primary cause of enhanced emotions (Zeelenberg et al., 2002). Alternatively, choosing to switch may make participants more likely to engage in counterfactual thinking, comparing actual outcomes with "what might have been," because outcomes associated with defaults may serve as a salient reference point (Kahneman et al., 1991). Future studies could test these possibilities by manipulating factors such as whether the participants themselves or another person makes the stay/switch choices.

The default bias can take many different forms depending on the nature of the default (e.g., to remain silent/inactive, to accept an assigned gambling card, or to conform to social norms). Whether or not other forms of default bias can be explained by the mechanisms identified here remains to be fully explored. However, recent fMRI research investigating a different variant of the default bias suggests that distinct mechanisms may underlie some different forms. Fleming et al. (2010) used a paradigm in which participants made a perceptual decision as to whether a ball fell within a tramline or outside it. On different trials, one of the options (in/out) was assigned as the default. Accepting it required no active response (continue to depress a key), whereas rejecting it required an overt action. Results showed that participants were more likely to accept the default when the perceptual decision was difficult but not when it was easy. In addition, rejecting the default on difficult, but not on easy, trials activated the subthalamic nucleus, a region implicated in overcoming response suppression and initiating an overt action (Isoda and Hikosaka, 2008). The discrepancies between the results of this study and our own might be explained by the different experimental designs. The paradigm of Fleming et al. (2010) minimized the influence of psychological variables such as loss aversion, included no immediate monetary feedback during the task, and required an active response on switch trials only. In contrast, our paradigm was optimized to investigate the role of emotions in the default bias by presenting monetary feedback after stay or switch choices on each trial and switch and stay options both required an active buttonpress response, matched for execution time.

In conclusion, our results identify the neural mechanisms contributing to the default inertia. These include an anticipatory somatic signal in the insula as a potential mechanism for loss aversion and a ventral striatal mechanism associated with default selection encompassing the same area as winning, implying that selecting the default might be rewarding in itself. These effects were found in the absence of switching cost (e.g., increased effort) and social influences (e.g., conformity to default social norms). While the functional basis of the default preference is still open to debate, our study highlights the key role of emotions in mediating this bias, illustrating that the power of default preference in both policy-making as well as more routine everyday behaviors is driven by basic emotional systems.

\section{References}

Anderson CJ (2003) The psychology of doing nothing: forms of decision avoidance result from reason and emotion. Psychol Bull 129:139-167.

Bechara A, Damasio H, Tranel D, Damasio AR (1997) Deciding advantageously before knowing the advantageous strategy. Science 275:1293-1295.

Buchel C, Morris J, Dolan RJ, Friston KJ (1998) Brain systems mediating aversive conditioning: an event-related fMRI study. Neuron 20:947-957.

Clark L, Bechara A, Damasio H, Aitken MR, Sahakian BJ, Robbins TW (2008) Differential effects of insular and ventromedial prefrontal cortex lesions on risky decision-making. Brain 131:1311-1322.

Craig AD (2009) How do you feel-now? The anterior insula and human awareness. Nat Rev Neurosci 10:59-70.

Critchley HD, Elliott R, Mathias CJ, Dolan RJ (2000) Neural activity relating to generation and representation of galvanic skin conductance 
responses: a functional magnetic resonance imaging study. J Neurosci 20:3033-3040.

Dunn B, Galton H, Morgan R, Evans D, Oliver C, Meyer M, Cusack R, Lawrence A, Dalgliesh T (2010) Listening to your heart: how interoception shapes emotion experience and intuitive decision making. Psychol Sci, in press.

Fleming SM, Thomas CL, Dolan RJ (2010) Overcoming status quo bias in the human brain. Proc Natl Acad Sci U S A 107:6005-6009.

Fliessbach K, Weber B, Trautner P, Dohmen T, Sunde U, Elger CE, Falk A (2007) Social comparison affects reward-related brain activity in the human ventral striatum. Science 318:1305-1308.

Friston KJ, Penny WD, Glaser DE (2005) Conjunction revisited. Neuroimage 25:661-667.

Isoda M, Hikosaka O (2008) Role for subthalamic nucleus neurons in switching from automatic to controlled eye movement. J Neurosci 28:7209-7218.

Johnson EJ, Goldstein D (2003) Medicine. Do defaults save lives? Science 302:1338-1339.

Kahneman D, Miller D (1986) Norm theory: comparing reality to its alternatives. Psychol Rev 93:136-153.

Kahneman D, Knetsch J, Thaler R (1991) Anomalies: the endowment effect, loss aversion, and status quo bias. J Econ Perspect 5:193-206.

Knutson B, Adams CM, Fong GW, Hommer D (2001) Anticipation of increasing monetary reward selectively recruits nucleus accumbens. J Neurosci 21:RC159.

Koyama T, McHaffie JG, Laurienti PJ, Coghill RC (2005) The subjective experience of pain: where expectations become reality. Proc Natl Acad Sci U S A 102:12950-12955.

Nieuwenhuis S, Heslenfeld DJ, von Geusau NJ, Mars RB, Holroyd CB, Yeung N (2005) Activity in human reward-sensitive brain areas is strongly context dependent. Neuroimage 25:1302-1309.
Nitschke JB, Dixon GE, Sarinopoulos I, Short SJ, Cohen JD, Smith EE, Kosslyn SM, Rose RM, Davidson RJ (2006) Altering expectancy dampens neural response to aversive taste in primary taste cortex. Nat Neurosci 9:435-442.

O’Doherty J, Dayan P, Schultz J, Deichmann R, Friston K, Dolan RJ (2004) Dissociable roles of ventral and dorsal striatum in instrumental conditioning. Science 304:452-454.

Paulus MP, Rogalsky C, Simmons A, Feinstein JS, Stein MB (2003) Increased activation in the right insula during risk-taking decision making is related to harm avoidance and neuroticism. Neuroimage 19:1439-1448.

Preuschoff K, Quartz SR, Bossaerts P (2008) Human insula activation reflects risk prediction errors as well as risk. J Neurosci 28:2745-2752.

Samuelson W, Zeckhauser R, J (1988) Status quo bias in decision making. J Risk Uncertainty 1:7-59.

Seymour B, O’Doherty JP, Dayan P, Koltzenburg M, Jones AK, Dolan RJ, Friston KJ, Frackowiak RS (2004) Temporal difference models describe higher-order learning in humans. Nature 429:664-667.

Sharot T, Riccardi AM, Raio CM, Phelps EA (2007) Neural mechanisms mediating optimism bias. Nature 450:102-105.

Sharot T, De Martino B, Dolan RJ (2009) How choice reveals and shapes expected hedonic outcome. J Neurosci 29:3760-3765.

Tom SM, Fox CR, Trepel C, Poldrack RA (2007) The neural basis of loss aversion in decision-making under risk. Science 315:515-518.

Tzourio-Mazoyer N, Landeau B, Papathanassiou D, Crivello F, Etard O, Delcroix N, Mazoyer B, Joliot M (2002) Automated anatomical labeling of activations in SPM using a macroscopic anatomical parcellation of the MNI MRI single-subject brain. Neuroimage 15:273-289.

Zeelenberg M, van de Bos K, van Dijk E, Pieters R (2002) The inaction effect in the psychology of regret. J Pers Soc Psychol 82:314-327. 